\title{
Organ donation - are we prepared for the future challenges?
}

${ }^{1} \mathrm{~S}$ Christie, ${ }^{2} \mathrm{I}$ Mellor ${ }^{2,3} \mathrm{~S}$ Cole

${ }^{1}$ Fellow in Intensive Care Medicine; ${ }^{2}$ Consultant in Anaesthesia and Intensive Care Medicine, Ninewells Hospital and Medical School, Dundee, Scotland, UK; ${ }^{3}$ Member, Academy of Medical Royal Colleges Donation Ethics Committee

This review is based in part on Dr Cole's lecture at the RCPE St Andrew's Day Festival Symposium held in Edinburgh on I and 2 December 201 I

KEYWORDS Organ donation, transplantation, donation after circulatory death, donation after brainstem death

DECLARATION OF INTERESTS Dr Christie and Dr Mellor have no conflicts of interest to declare. $\mathrm{Dr}$ Cole is Joint Regional Clinical Lead for Organ Donation and Co-chair NHS Tayside Donation Committee

It is now over 50 years since Sir Michael Woodruff performed the UK's first successful kidney transplant in Edinburgh in 1960.Transplantation surgery has continually evolved over the intervening years, providing life-saving opportunities for many patients. However, despite the technical advances in treatment available and improved medical management of underlying conditions, some patients may not survive due to the shortage of organs available for donation. There are currently 7,762 patients in the UK awaiting transplantation, but three patients on average will die every day due to the chronic lack of available organs.'

The UK compares unfavourably with other countries in terms of the number of deceased organ donors per million population (pmp). We have approximately 14 pmp while Spain for example has almost $35 \mathrm{pmp}$. The Spanish model has often been held up as the ideal; not always the case though, this has been achieved through the introduction and widespread adoption of a number of specific polices and protocols over a number of years.

In an effort to improve UK organ donation rates the Organ Donation Task Force was formed by the Department of Health in 2006 and published a report containing 14 recommendations in 2008 . The task force identified barriers to organ donation and recommended actions to address them. The report stated that rates could be increased by $50 \%$ by $2013 .{ }^{2}$ This is an ambitious plan that combines several strategies for optimising support for organ donation: on a national level the aim is to increase registration on the Organ Donor Register; on a local level policies would be implemented, driven by clinical leads within individual hospitals. One of the main strategic goals is to raise awareness among National Health Service (NHS) executives, the medical profession and the general public about the severity of the problem and to make consideration of donation a routine part of end-of-life care planning. These recommendations were accepted in full by the devolved health administrations.

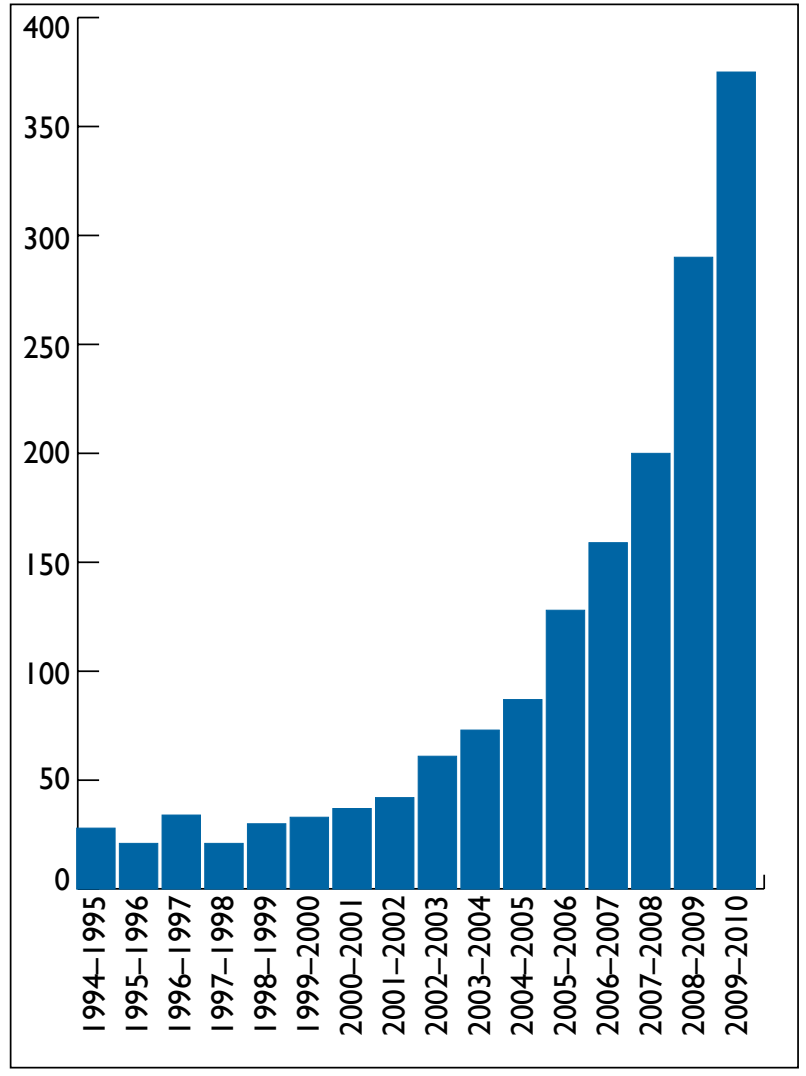

FIGURE I Number of patients in the UK donating organs following circulatory death.

\section{ORGAN DONATION IN SCOTLAND}

When surveyed, approximately $87 \%$ of the UK population expressed support for organ donation ${ }^{3}$ however only $37 \%$ of the Scottish population are on the Organ Donor Register (July 20 I0).There have been recent improvements in raising awareness of this issue, and there has been an increase, particularly in young adults (aged 16-25) in the number of people registering their choice to donate their organs. Despite these advances, Scotland continues to lag behind other European countries and there is an ongoing 
shortage of organs from deceased donors each year. NHS Scotland has recommended that organ donation routinely forms part of the discussions surrounding end-of-life care, when appropriate. ${ }^{4}$

Over $95 \%$ of the Scottish population would be happy to receive an organ transplant if it was required. ${ }^{3} \mathrm{~A}$ valid ethical argument could be made that if someone has indicated that they would accept an organ from a deceased donor, they should be prepared to donate their own organs after death. This argument moves inevitably towards a discussion of a system of presumed consent, where individuals would actively have to opt out of organ donation. This topic is currently the subject of a white paper in the Welsh Assembly.

\section{ORGAN DONATION FOLLOWING BRAINSTEM DEATH AND CIRCULATORY DEATH IN THE UK}

Over the past ten years the number of cases of organ donation following brainstem death (DBD) has remained relatively static with approximately 40-60 patients per year in Scotland. ${ }^{5}$ Improvements in neurosurgery and intensive care combined with robust public health campaigns on road safety mean that future substantial increases in the number of DBD patients are unlikely. The UK as a whole has a much lower potential number of brainstem dead patients than other countries, notably Spain.This is in part due to the public health improvements noted above but also because of the differences in admission thresholds to intensive care and cultural attitudes towards end-of-life care. In Spain there are more intensive care beds and it is not uncommon to admit patients who have suffered severe strokes to provide support and allow brainstem death to occur over a period of days or weeks as part of end-of-life care. This would generally be considered unethical in the UK.

In recent years there has been a ten-fold increase in the number of patients donating organs following circulatory death (DCD) and DCD now accounts for $35 \%$ of all deceased donations in the UK. ${ }^{5}$

For patients receiving a kidney transplant from a donor following either DCD or DBD, the outcome is similar. Liver, lung and pancreas transplants have acceptable outcomes as a result of the technical advances in organ retrieval after circulatory death. ${ }^{5}$ Heart retrieval is not currently undertaken following DCD in the UK. However, the UK Donation Ethics Committee (UKDEC) of the Academy of Medical Royal Colleges has investigated the ethical implications of this issue for both adults and children.There are a number of cardiac transplant centres in the UK prepared to implement such a heart transplantation programme following regulatory approval.

The average number of solid organs retrieved per donor is 4.I for DBD compared with 2.I for DCD in Scotland
$(2009 / 10) .{ }^{5}$ Donation after circulatory death is currently regarded as the route with the greatest potential for increasing the number of available organs for transplantation. It is not a new process, the world's first heart transplant in South Africa in 1967 was carried out following the circulatory death of the donor.

The drive to increase the number of organ donations following DCD has been challenging for many intensive care physicians who are working in difficult clinical conditions at a time when there has not always been clear ethical and legal guidance. This has improved with legal clarification from both the UK and Scottish Governments of the relevant legislation. The Adults with Incapacity (Scotland) Act is relevant prior to death and the Human Tissue (Scotland) Act ${ }^{6,7}$ which applies after death.

The UKDEC recently published an ethical framework for controlled donation after circulatory death. ${ }^{8}$ It has recommended that the existing practice for the diagnosis of brainstem death continues to be followed: two senior clinicians must document the futility of continuing lifesustaining treatment. They also recommend that this discussion remains completely separate from any consideration for the potential to donate organs. Only after a clearly documented note of futility and planned withdrawal of life-sustaining treatment has been made will the organ donation register be checked and a request made in collaboration with a specialist nurse.?

Managing the organ donation process following $D C D$ requires considerable resources and experience. It is based on two main principles: that organ donation is regarded as a routine part of end-of-life care planning, and when it has been agreed that organ donation is in the patient's interests, the ethical imperative is to ensure the most successful outcome for that donation.

Excellent communication skills are required by the medical team: the patient's family must be able to make informed decisions regarding donation at a time of extreme emotional distress. Everyone involved in the process must be aware that organ retrieval may not be possible if the warm ischaemic time becomes prolonged and death does not occur within a two to four hour window following withdrawal of life-sustaining treatment. They also need to be aware that the timing of the procedure will be driven by the identification of suitable recipients and the availability of the retrieval team. Withdrawal of life-sustaining treatment will not begin until the retrieval team are on-site, in theatre and ready to proceed. This may be many hours from the original decisions about the futility of continuing treatment and end-of-life options.

Ongoing support and adjustment of the patient's physiology will be required in the interim in order to ensure optimal organ function and to allow enough time 
for the retrieval team to arrive. This is time consuming and requires ongoing close attention to detail.

Despite the publication of consensus guidelines there are still differences of opinion within the intensive care community regarding the management of controlled circulatory death donation. The debate about how to withdraw life-sustaining treatment continues. Some clinicians argue that the same procedures for end-of-life care should be followed regardless of the plans to donate. The counter argument is that optimising the ability to donate takes precedence, and this can potentially be achieved in the required timeframe only if the patient's airway and sedation regime are altered to make the end-of-life process shorter, i.e. stopping rather than weaning cardiovascular and respiratory support and moving to extubate the patient's trachea. Regardless of these differences of opinion it is essential that each intensive care unit has an established process to allow DCD to proceed. If members of the medical team have ethical concerns surrounding this decision, a colleague can take on the management of the case, in the same way that individuals may opt out of providing anaesthesia in cases of termination of pregnancy or blood treatment for Jehovah's Witnesses.

\section{EMERGENCY DEPARTMENTS}

A source for potential increases in organ donation rates that has been relatively unrecognised until recently is the emergency department (ED). Significant numbers of patients die in these areas, particularly from catastrophic pathology deemed to be irreversible. The intensive care unit physicians have historically allowed patient admissions only when there are treatment options available to improve the outcome. As the public become increasingly aware of end-of-life decisions and sign up to the organ donor register, there will be an expectation that their previously expressed wishes are respected. This creates a new challenge for the intensive care team who will be required to manage these patients to enable donation to proceed if possible.

A number of important issues will need to be addressed in the future. Senior clinicians for example should make decisions regarding the futility of continuing treatment but this may not always be possible, particularly out-ofhours when they are not available. There is an inherent danger in making decisions rapidly in the ED when the patient's condition may continue to evolve. Managing the family's expectations at a time when they are traumatised and in shock must be handled carefully, including explanations that not all patients will die when supportive treatment is withdrawn. A time may come when decisions need to be made regarding transferring a potential donor to another unit with the capacity to provide the required care. The emotional issues must not be underestimated.
Perhaps one of the greatest challenges we have yet to face are the implications for critical care resources of increasing donation rates. Managing the care of potential organ donors is best achieved within the intensive care unit where dedicated staff have the required skills and expertise. Quality of care decreases when staff and resources are stretched and the UK has not invested in large-scale expansions of intensive care units to meet the ambitious plans for organ donation. Many intensive care clinicians continue to question the ethical validity of potentially compromising the care of patients who could survive intensive care because their resources are stretched managing the potential organ donor. This raises the question of how to balance the benefits to the individual vs the benefit to society.

Organ donation and transplantation is one of the greatest success stories in medicine. Its ability to transform thousands of lives each year makes the ambitious targets for improving this field worth striving for. Increased public awareness of the positive results of organ donation means that many families will continue to act with generosity and a desire to fulfil the wishes of their loved ones. The challenge we face is providing this opportunity to an increasing number of patients at a time of very limited expansion in resources.

Organ donation must become an established and usual part of end-of-life care. In order to make this happen, we must address the needs of the critical care community to take on this workload as well as the attitudes of society and the healthcare professions.

\section{REFERENCES}

I NHS Blodd and Transplant. Organ donation [Internet]; c 20II [cited 20I I Oct 2]. Available from: www.uktransplant.org.uk

2 Department of Health. Organs for transplants [Internet]. London: Department of Health; 2008 [cited 2011 Oct 2]. Available from: http://www.dh.gov.uk/en/Publicationsandstatistics/Publications/ PublicationsPolicyAndGuidance/DH_082 I22.

3 ICM/Endemol/BBC. Survey of General public - UK study of 1027 participants. London: BBC; 2005.

4 Scottish Government. Living and dying well [Internet]. Edinburgh: NHS Scotland; 2008 [cited 20II Feb 17]. Available from: http:// www.scotland.gov.uk/Resource/Doc/239823/0066I55.pdf

5 British Transplantation Society, Intensive Care Society, Department of Health. Donation after circulatory death. Report of consensus meeting [Internet]. London: Department of Health; 2010 [cited 20II Feb I7]. Available at: http://www.ics.ac.uk/intensive_care_professional/ standards_and_guidelines/ded

6 The Scottish Government. Human Tissue Act Scotland 2006 [Internet]. Edinburgh: The Scottish Government; 2006 (cited 20II Feb 22]. Available from: http://www.legislation.gov.uk/asp/2006/4/contents

7 The Scottish Government. Adults with Incapacity (Scotland) Act 2000 Part 5 Section 47 [Internet]. Edinburgh:The Scottish Government; 2006 (cited 20II Feb 22]. Available from: http://www.scotland.gov. uk/Publications/2008/06/I3 I I4II7/0

8 UK Donation Ethics Committee, Academy of Medical Royal Colleges. An ethical framework for controlled donation after circulatory death consultation [Internet]. London: Academy of Medical Royal Colleges; 20II [cited 20II Feb 22]. Available from: http://www. aomrc.org.uk/committees/uk-donation-ethics-committee.html 